\title{
Rigorous pointwise approximations for invariant densities of non-uniformly expanding maps
}

\author{
WAEL BAHSOUN $\dagger$, CHRISTOPHER BOSE $\ddagger$ and YUEJIAO DUAN $\dagger$ \\ $\dagger$ Department of Mathematical Sciences, Loughborough University, Loughborough, \\ Leicestershire, LE11 3TU, UK \\ (e-mail: W.Bahsoun@lboro.ac.uk,Y.Duan@lboro.ac.uk) \\ $\$$ Department of Mathematics and Statistics, University of Victoria, \\ PO Box 3045 STN CSC, Victoria, BC, V8W 3R4, Canada \\ (e-mail: cbose@uvic.ca)
}

(Received 16 January 2013 and accepted in revised form 31 August 2013)

\begin{abstract}
We use an Ulam-type discretization scheme to provide pointwise approximations for invariant densities of interval maps with a neutral fixed point. We prove that the approximate invariant density converges pointwise to the true density at a rate $C^{*}$. $(\ln m) / m$, where $C^{*}$ is a computable fixed constant and $m^{-1}$ is the mesh size of the discretization.
\end{abstract}

\section{Introduction}

Ulam-type discretization schemes provide rigorous approximations for dynamical invariants. Moreover, such discretizations are easily implementable on a computer. In [18] it was shown that the original Ulam method [23] is remarkably successful in approximating isolated spectrum of transfer operators associated with piecewise expanding maps of the interval. In particular, it was shown that this method provides rigorous approximations in the $L^{1}$-norm for invariant densities of Lasota-Yorke maps (see [18] and references therein). This method has been also successful when dealing with multi-dimensional piecewise expanding maps [20], and partially successful§ in providing rigorous approximations for certain uniformly hyperbolic systems $[\mathbf{1 0}, \mathbf{1 1}]$. Recently, Blank [5] and Murray [21] independently succeeded in applying the pure Ulam method in a non-uniformly hyperbolic setting. They obtained approximations in the $L^{1}$-norm for invariant densities of certain

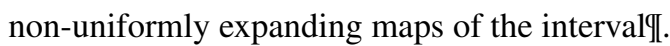

Although $L^{1}$ approximations provide significant information about the long-term statistics of the underlying system, they are not helpful when dealing with rare events 
in dynamical systems. In fact, when studying rare events in dynamical systems $[\mathbf{1 , 1 5}]$ one often obtains probabilistic laws that depend on pointwise information from the invariant density of the system. In particular, extreme value laws of interval maps with a neutral fixed point depend pointwise on the invariant density of the map [13].

Statistical properties of non-uniformly expanding maps were studied by Pianigiani [22], who first proved existence of invariant densities of such maps. Later, it was independently proved in $[\mathbf{1 4}, \mathbf{1 9}, \mathbf{2 4}]$ that such maps exhibit polynomial decay of correlations. The slow mixing behaviour that such maps exhibit has made them good testing tools for real and difficult physical problems.

The difficulty in obtaining pointwise approximations for invariant densities of interval maps with a neutral fixed point is twofold. Firstly, the transfer operator associated with such maps does not have a spectral gap in a classical Banach space. Therefore, powerful perturbation results $[\mathbf{1 6}] \dagger$ are not directly available in this setting. Secondly, invariant densities of such maps are not $L^{\infty}$ functions. Consequently, to provide pointwise approximation of such densities, one should first measure the approximations in a 'properly weighted' $L^{\infty}$-norm.

In this paper we use a piecewise linear Ulam-type discretization scheme to provide pointwise approximations for invariant densities of non-uniformly expanding interval maps. Our main result is stated in Corollary 3.2. For $x \in(0,1]$ we prove that the approximate invariant density converges pointwise to the true density at a rate $C^{*} / x^{1+\alpha}$. $(\ln m) / m$, where $C^{*}$ is a computable fixed constant, $\alpha \in(0,1)$ is a fixed constant, and $m^{-1}$ is the mesh size of the discretization. To overcome the spectral difficulties and the unboundedness of the densities which we discussed above, we first induce the map and obtain a uniformly piecewise, expanding and onto map. Then we perform our discretization on the induced space. After that we pull back, both the invariant density and the approximate one to the full space and measure their difference in a weighted $L^{\infty}$ norm. Full details of our strategy are given in $\$ 3.2$.

In $\S 2$, we recall results on uniformly piecewise expanding and onto maps. Moreover, we introduce our discretization scheme and recall results about uniform approximations for invariant densities of uniformly piecewise expanding and onto maps. In $\S 3$, we introduce our non-uniformly expanding system, set up our strategy, and state our main results, Theorem 3.1 and Corollary 3.2. Section 4 contains technical lemmas and the proof of Theorem 3.1. Section 5 presents an algorithm based on the result of Corollary 3.2 and discusses its feasibility.

\section{Preliminaries}

2.1. A piecewise expanding system. Let $(\Delta, \mathfrak{B}, \hat{\lambda})$ denote the measure space where $\Delta$ is a closed interval, $\mathfrak{B}$ is Borel $\sigma$-algebra and $\hat{\lambda}$ is normalized Lebesgue measure on $\Delta$. Let $\hat{T}: \Delta \rightarrow \Delta$ be a measurable transformation. We assume that there exists a countable partition $\mathcal{P}$ of $\Delta$, which consists of a sequence of intervals, $\mathcal{P}=\left\{I_{i}\right\}_{i=0}^{\infty}$, such that:

(1) for each $i=1, \ldots, \infty, \hat{T}_{i}:=\hat{T}_{\mid I_{i}}$ is monotone, $C^{2}$, and it extends to a $C^{2}$ function on $\bar{I}_{i}$;

$\dagger$ See also [12] for another perturbation result. 
(2) $\quad \hat{T}_{i}\left(I_{i}\right)=\Delta$, i.e. for each $i=1, \ldots, \infty, \hat{T}_{i}$ is onto;

(3) there exists a constant $D>0$ such that $\sup _{i} \sup _{x \in I_{i}}\left(\left|\hat{T}^{\prime \prime}(x)\right| /\left(\hat{T}^{\prime}(x)\right)^{2}\right) \leq D$;

(4) there exists a number $\gamma$ such that $1 /\left|\hat{T}_{i}^{\prime}\right| \leq \gamma<1$.

Let $\hat{\mathcal{L}}: L^{1} \rightarrow L^{1}$ denote the transfer operator (Perron-Frobenius) $[\mathbf{4 , 8}]$ associated to $\hat{T}$ :

$$
\hat{\mathcal{L}} f(x)=\sum_{y=\hat{T}^{-1} x} \frac{f(y)}{\left|\hat{T}^{\prime}(y)\right|} .
$$

Under the above assumptions, among other ergodic properties, it is well known that (see, for instance, [7]) $\hat{T}$ admits a unique invariant density $\hat{f}$, i.e. $\hat{\mathcal{L}} \hat{f}=\hat{f}$. Moreover, $\hat{\mathcal{L}}$ admits a spectral gap when acting on the space of Lipschitz continuous functions over $\Delta[2] \dagger$. We will denote by $B V(\Delta)$ the space of functions of bounded variation defined on the interval $\Delta$. Set $\|\cdot\|_{B V(\Delta)}:=V_{\Delta}+\|\cdot\|_{1, \Delta}$, where $V_{\Delta}$ denotes the one-dimensional variation over $\Delta$. Then it is well known that $\left(B V(\Delta),\|\cdot\|_{B V(\Delta)}\right)$ is a Banach space and $\hat{\mathcal{L}}$ satisfies the following inequality (see, for instance, [22]): there exists a constant $C_{\mathrm{LY}}>0$ such that, for any $f \in B V(\Delta)$, we have

$$
V_{\Delta} \hat{\mathcal{L}} f \leq \gamma V_{\Delta} f+C_{\mathrm{LY}}\|f\|_{1, \Delta} .
$$

Inequality (2.1) is called the Lasota-Yorke inequality.

2.2. Markov discretization. We now introduce a discretization scheme which enables us to obtain rigorous uniform approximation of $\hat{f}$, the invariant density of $\hat{T}$. We use a piecewise linear approximation which was introduced by Ding and Li [9]. Let $\eta=\left\{c_{i}\right\}_{i=0}^{m}$ be a partition of $\Delta$ into intervals. Since uniform partitions are the first choice for numerical work, we set $c_{i}-c_{i-1}=|\Delta| / m$, where $|\Delta|$ is the length of $\Delta$. Everything we do can be easily modified for non-uniform partitions with only minor notational changes. Let

$$
\varphi_{i}=\chi_{\left[c_{i-1}, c_{i}\right]} \quad \text { and } \quad \phi_{i}(x)=\frac{m}{|\Delta|} \int_{0}^{x} \varphi_{i} d \lambda .
$$

Let $\psi_{i}$ denote a set of hat functions over $\eta$ :

$$
\psi_{0}:=\left(1-\phi_{1}\right), \quad \psi_{m}:=\phi_{m} \quad \text { and for } i=1, \ldots, m-1, \quad \psi_{i}:=\left(\phi_{i}-\phi_{i+1}\right) .
$$

For $f \in L^{1}$, we set $I_{i}:=\left[c_{i-1}, c_{i}\right]$ and

$$
f_{i}:=\frac{m}{|\Delta|} \int_{I_{i}} f d x, \quad i=1,2, \ldots, m
$$

the average of $f$ over the associated partition cell. For $f \in L^{1}$ we set

$$
Q_{m} f:=f_{1} \psi_{0}+\sum_{i=1}^{m-1} \frac{f_{i}+f_{i+1}}{2} \psi_{i}+f_{m} \psi_{m} .
$$

Obviously, the operator $Q_{m}$ retains good stochastic properties, i.e.:

- $\quad$ for $f \geq 0, Q_{m} f \geq 0$;

- $\int Q_{m} f=\int f$.

$\dagger$ In [2], a Lasota-Yorke inequality was obtained for Markov interval maps with a finite partition. The proof carries over for piecewise onto maps with a countable number of branches satisfying the assumptions of $\S 2.1$. 
We now define a piecewise linear Markov discretization of $\hat{\mathcal{L}}$ by

$$
\mathbb{P}_{m}:=Q_{m} \circ \hat{\mathcal{L}}
$$

Notice that $\mathbb{P}_{m}$ is a finite-rank Markov operator whose range is contained in the space of continuous, piecewise linear functions with respect to $\eta$. The matrix representation of $\mathbb{P}_{m}$ restricted to this finite-dimensional space and with respect to the basis $\left\{\psi_{i}\right\}$ is a (row) stochastic matrix. By the Perron-Frobenius theorem for stochastic matrices [17], $\mathbb{P}_{m}$ has a left invariant density $\hat{f}_{m}$, i.e.

$$
\hat{f}_{m}=\hat{f}_{m} \mathbb{P}_{m}
$$

The following theorem was proved in [2].

THEOREM 2.1. There exists a computable constant $\hat{C}$ such that, for any $m \in \mathbb{N}$,

$$
\left\|\hat{f}-\hat{f}_{m}\right\|_{\infty} \leq \hat{C} \frac{\ln m}{m} .
$$

Remark 2.2. We recall that in [2] it was shown that the constant $\hat{C}$, which is independent of $m$, can be computed explicitly.

3. Pointwise approximations for invariant densities of maps with a neutral fixed point

3.1. The non-uniformly expanding system. Let $I=[0,1]$ be the unit interval, $\lambda$ be the Lebesgue measure on $[0,1]$. Let $T: I \rightarrow I$ be a piecewise smooth map with two branches. We assume that:

- $\quad T(0)=0$ and there is an $x_{0} \in(0,1)$ such that $T_{1}=\left.T\right|_{\left[0, x_{0}\right)}, T_{2}=\left.T\right|_{\left[x_{0}, 1\right]}$ and $T_{1}:\left[0, x_{0}\right) \stackrel{\text { onto }}{\longrightarrow}[0,1), T_{2}:\left[x_{0}, 1\right] \stackrel{\text { onto }}{\longrightarrow}[0,1]$;

- $\quad T_{1}$ is $C^{1}$ on $\left[0, x_{0}\right], T_{1}$ is $C^{2}$ on $\left(0, x_{0}\right]$ and $T_{2}$ is $C^{2}$ on $\left[x_{0}, 1\right]$;

- $\quad T^{\prime}(0)=1$ and $T^{\prime}(x)>1$ for $x \in\left(0, x_{0}\right) ;\left|T^{\prime}(x)\right| \geq \beta>1$ for $x \in\left(x_{0}, 1\right)$;

- $\quad T_{1}$ and $T_{1}^{\prime}$ have the form

$$
\begin{gathered}
T_{1}(x)=x+x^{1+\alpha}+x^{1+\alpha} \delta_{0}(x), \\
T_{1}^{\prime}(x)=1+(1+\alpha) x^{\alpha}+x^{\alpha} \delta_{1}(x),
\end{gathered}
$$

where, $0<\alpha<1$ and $\delta_{i}(x) \rightarrow 0$ as $x \rightarrow 0$ for $i=0,1$ with $\delta_{0}^{\prime}(x) \geq 0$.

It is well known that $T$ admits a unique invariant density $f^{*}[\mathbf{1 4}, \mathbf{1 9}, \mathbf{2 2}, \mathbf{2 4}]$ and the system $\left(I, T, f^{*} \cdot \lambda\right)$ exhibits a polynomial mixing rate $[\mathbf{1 4}, \mathbf{1 9}, \mathbf{2 4}]$. Moreover, it is well known $[14,19,24]$ that the $T$-invariant density, $f^{*}$, is not an $L^{\infty}$-function. In particular, near $x=0, f^{*}(x)$ behaves like $x^{-\alpha}$. Despite this difficulty, we will show that, for any $x \in(0,1]$, one can obtain rigorous pointwise approximation of $f^{*}(x)$.

3.2. Strategy and the statement of the main result. Recall that $\alpha \in(0,1)$. We first define a suitable Banach space that contains $f^{*}$. More precisely, let $\mathcal{B}$ denote the set of continuous functions on $(0,1]$ with the norm

$$
\|f\|_{\mathcal{B}}=\sup _{x \in(0,1]}\left|x^{1+\alpha} f(x)\right| .
$$


When equipped with the norm $\|\cdot\|_{\mathcal{B}}, \mathcal{B}$ is a Banach space $\dagger$. The fact that $f^{*} \in \mathcal{B}$ follows from [14, Lemma 3.3]. Our strategy for obtaining a pointwise approximation of $f^{*}$ consists of the following steps.

(1) We first induce $T$ on $\Delta \subset I$ and obtain a $\hat{T}$ which satisfies the assumptions of $\S 2.1$.

(2) On $\Delta$, we use Theorem 2.1 to say that $\hat{f}_{m}$, the invariant density of the discretized operator $\mathbb{P}_{m}:=Q_{m} \circ \hat{\mathcal{L}}$, defined in equation (2.3), provides a uniform approximation of $\hat{f}$ the $\hat{T}$-invariant density.

(3) Next we write $f^{*}$ in terms of $\hat{f}$, and define a function $f_{m}$ as the 'pullback' of $\hat{f}_{m}$.

(4) Finally, we use steps (2) and (3) to prove that $\left\|f^{*}-f_{m}\right\|_{\mathcal{B}} \leq C^{*}((\ln m) / m)$, and deduce a pointwise approximation of $f^{*}$.

3.2.1. The induced system We induce $T$ on $\Delta:=\left[x_{0}, 1\right]$. For $n \geq 0$ we define

$$
x_{n+1}=T_{1}^{-1}\left(x_{n}\right) \text {. }
$$

Set

$$
W_{0}:=\left(x_{0}, 1\right) \quad \text { and } \quad W_{n}:=\left(x_{n}, x_{n-1}\right), \quad n \geq 1 .
$$

For $n \geq 1$, we define

$$
Z_{n}:=T_{2}^{-1}\left(W_{n-1}\right)
$$

Then we define the induced map $\hat{T}: \Delta \rightarrow \Delta$ by

$$
\hat{T}(x)=T^{n}(x) \text { for } x \in Z_{n} .
$$

Observe that

$$
T\left(Z_{n}\right)=W_{n-1} \quad \text { and } \quad \tau_{Z_{n}}=n,
$$

where $\tau_{Z_{n}}$ is the first return time of $Z_{n}$ to $\Delta$. An example of the map $T$ and its induced counterpart $\hat{T}$ are shown in Figures 1 and 2 respectively. It is well known (see, for instance, [24]) that the $\hat{T}$ defined in (3.1) satisfies the assumptions of $\S 2.1$, and, by Theorem 2.1, one can obtain a rigorous uniform approximation of its invariant density $\hat{f}$. Moreover, by [3, Lemma 3.3], $f^{*}$, the invariant density of $T$, can be written in terms of $\hat{f}$ :

$$
f^{*}(x)= \begin{cases}c_{\tau} \hat{f}(x) & \text { for } x \in \Delta, \\ c_{\tau} \sum_{n=1}^{\infty}\left(\frac{\hat{f}\left(T_{2}^{-1} T_{1}^{-(n-1)} x\right)}{\left|D T^{(n)}\left(T_{2}^{-1} T_{1}^{-(n-1)} x\right)\right|}\right) & \text { for } x \in I \backslash \Delta,\end{cases}
$$

where $\hat{f}$ is the $\hat{T}$-invariant density, $c_{\tau}^{-1}=\sum_{k=1}^{\infty} \tau_{Z_{k}} \hat{\mu}\left(Z_{k}\right)$, and $\hat{\mu}=\hat{f} \cdot \hat{\lambda}$.

\subsubsection{The approximate density and the statement of the main result. Set}

$$
f_{m}(x) \stackrel{\text { def }}{=} \begin{cases}c_{\tau, m} \hat{f}_{m}(x) & \text { for } x \in \Delta, \\ c_{\tau, m} \sum_{n=1}^{\infty}\left(\frac{\hat{f}_{m}\left(T_{2}^{-1} T_{1}^{-(n-1)} x\right)}{\left|D T^{(n)}\left(T_{2}^{-1} T_{1}^{-(n-1)} x\right)\right|}\right) & \text { for } x \in I \backslash \Delta,\end{cases}
$$

$\dagger$ In what follows, we only use the metric properties of $\mathcal{B}$. In particular, the completeness of $\mathcal{B}$ is not needed in our proofs. 


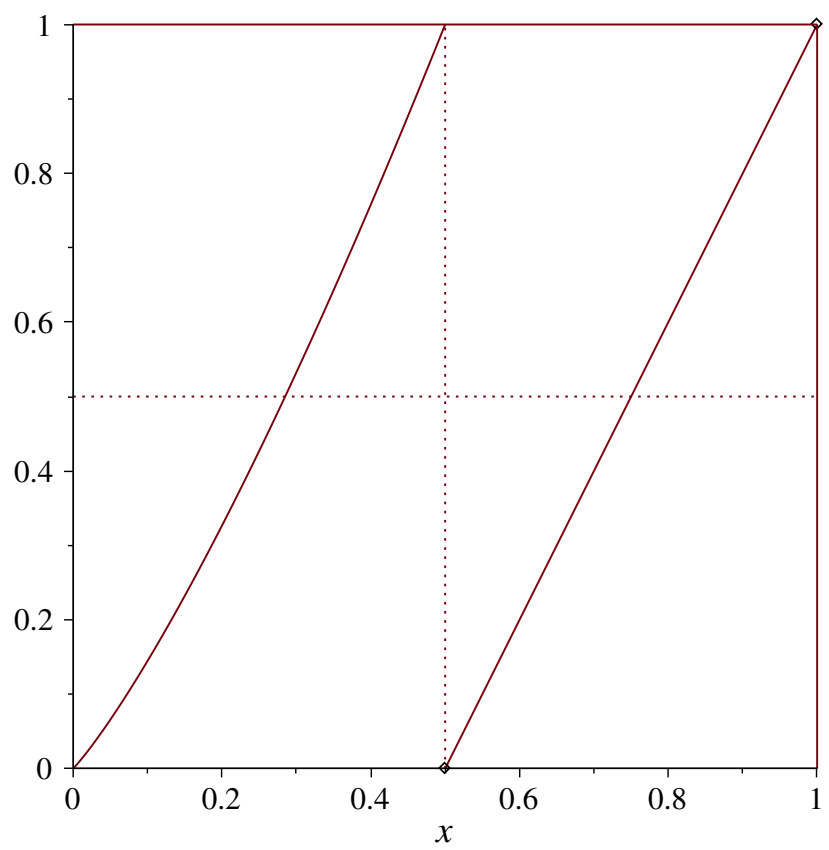

FIGURE 1. A typical example of a map $T$ which belongs to the family defined in $\S 3.1$.

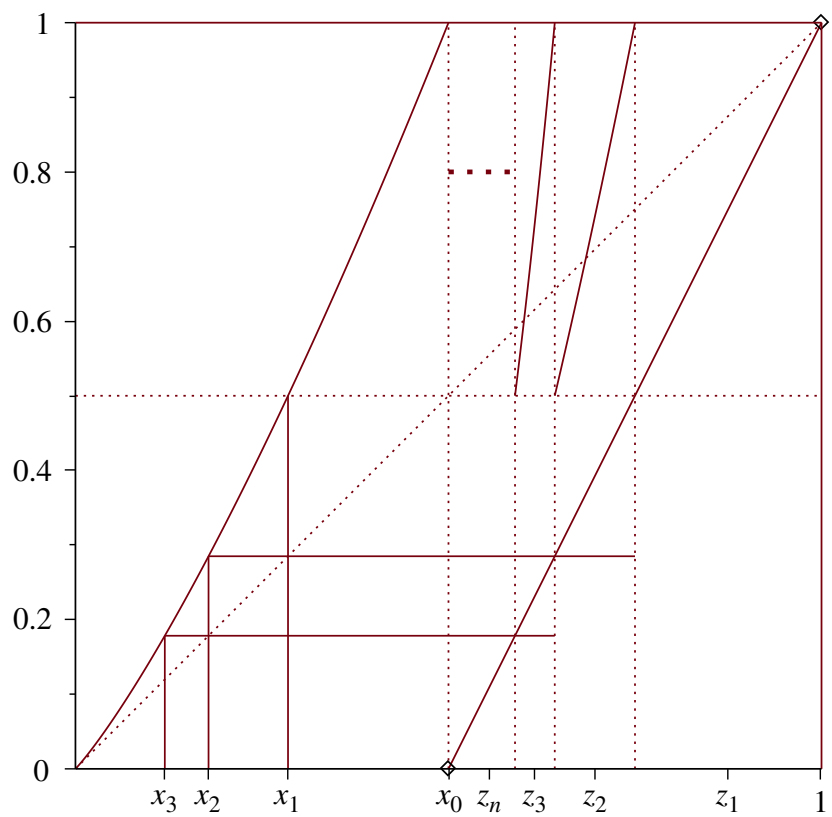

FIGURE 2. This figure shows the induced map $\hat{T}$ corresponding to the map $T$ of Figure 1. 
where $\hat{f}_{m}=\mathbb{P}_{m} \hat{f}_{m}$, and $\mathbb{P}_{m}$ is the Markov discretization of $\hat{\mathcal{L}}$ defined in (2.3), $c_{\tau, m}^{-1}=$ $\sum_{k=1}^{\infty} \tau_{Z_{k}} \hat{\mu}_{m}\left(Z_{k}\right)$, and $\hat{\mu}_{m}=\hat{f}_{m} \cdot \hat{\lambda}$. The next result shows that the function $f_{m}$ defined in (3.3) provides a rigorous pointwise approximation of $f^{*}$.

THEOREM 3.1. For any $m \in \mathbb{N}$ we have

$$
\left\|f^{*}-f_{m}\right\|_{\mathcal{B}} \leq C^{*} \frac{\ln m}{m},
$$

where

$$
C^{*}=\hat{C}\left(1+\frac{x_{0}^{1+\alpha}}{\beta}+M(1+\alpha)\right) C_{4} ;
$$

in particular, $\hat{C}$ is the computable constant of Theorem 2.1,

$$
\begin{gathered}
M:=\frac{C_{1}^{1+\alpha} e^{2 C_{0} C_{1}^{2 \alpha}}}{\beta}, \\
C_{0}:=\frac{\alpha(1+\alpha)}{2}\left[1+2 \delta_{0}\left(x_{0}\right)+\delta_{0}^{2}\left(x_{0}\right)\right], \quad C_{1}:=\left(2\left[2^{1 / \alpha}-1\right]\right)^{1 / \alpha}, \\
C_{4}:=1+C_{3}\left(\frac{C_{\mathrm{LY}}}{1-\gamma}+\frac{1}{|\Delta|}\right), \quad C_{3}:=\frac{1}{\beta}+\frac{C_{2}}{\beta|\Delta|}\left(\alpha+\frac{2-\alpha}{1-\alpha}\right),
\end{gathered}
$$

and

$$
C_{2}=\frac{1-x_{0}}{x_{0}^{1+\alpha}} 2^{1+(1 / \alpha)}\left[2^{1 / \alpha}-1\right]^{1+(1 / \alpha)} .
$$

As a direct consequence of Theorem 3.1 we obtain the required pointwise approximation of $f^{*}$.

COROLlary 3.2. For any $x \in(0,1]$ we have

$$
\left|f^{*}(x)-f_{m}(x)\right| \leq \frac{C^{*}}{x^{1+\alpha}} \frac{\ln m}{m} .
$$

Proof. For $x \in(0,1]$, we have

$$
\begin{aligned}
\left|f^{*}(x)-f_{m}(x)\right| & =\frac{1}{x^{1+\alpha}}\left|x^{1+\alpha}\left(f^{*}(x)-f_{m}(x)\right)\right| \\
& \leq \frac{1}{x^{1+\alpha}}\left\|f^{*}-f_{m}\right\|_{\mathcal{B}} \leq \frac{1}{x^{1+\alpha}} C^{*} \frac{\ln m}{m} .
\end{aligned}
$$

\section{Proofs}

4.1. Technical lemmas. We first introduce notation of certain functions which appear in the proof of Theorem 3.1. For $x \in I \backslash \Delta$, set

$$
\begin{aligned}
& g(x):=\frac{\left(T_{1} x / x\right)^{1+\alpha}}{T_{1}^{\prime}(x)}, \\
& G_{1}(x):=\frac{x^{1+\alpha}}{\left|T_{2}^{\prime}\left(T_{2}^{-1} x\right)\right|} \quad \text { and for } n \geq 2, \quad G_{n}(x):=\frac{x^{1+\alpha}}{\left|D T^{(n)}\left(T_{2}^{-1} T_{1}^{-(n-1)} x\right)\right|} .
\end{aligned}
$$


LEMMA 4.1. For $x \in I \backslash \Delta$, we have

$$
\left[1+x^{\alpha}+x^{\alpha} \delta_{0}(x)\right]^{1+\alpha} \leq 1+(1+\alpha)\left[x^{\alpha}+x^{\alpha} \delta_{0}(x)\right]+\frac{\alpha(1+\alpha)}{2}\left[x^{\alpha}+x^{\alpha} \delta_{0}(x)\right]^{2} .
$$

Proof. Let

$$
\phi_{1}(x):=\left[1+x^{\alpha}+x^{\alpha} \delta_{0}(x)\right]^{1+\alpha}
$$

and

$$
\phi_{2}(x):=1+(1+\alpha)\left[x^{\alpha}+x^{\alpha} \delta_{0}(x)\right]+\frac{\alpha(1+\alpha)}{2}\left[x^{\alpha}+x^{\alpha} \delta_{0}(x)\right]^{2} .
$$

Note that $\phi_{1}(0)=\phi_{2}(0)=1$. Therefore, to prove the lemma, it is enough to prove that $\phi_{1}^{\prime}(x) \leq \phi_{2}^{\prime}(x)$. We have

$$
\begin{aligned}
& \phi_{1}^{\prime}(x)=(1+\alpha)(1+\xi(x))^{\alpha} \xi^{\prime}(x), \\
& \phi_{2}^{\prime}(x)=(1+\alpha)(1+\alpha \xi(x)) \xi^{\prime}(x),
\end{aligned}
$$

where $\xi(x):=x^{\alpha}+x^{\alpha} \delta_{0}(x) \geq 0 \dagger$. Notice that $\xi^{\prime}(x) \geq 0$. Thus, we only need to show that

$$
(1+\xi(x))^{\alpha} \leq(1+\alpha \xi(x)) .
$$

Indeed, (4.1) holds because $(1+\xi(0))^{\alpha}=(1+\alpha \xi(0))=1$ and

$$
\left[(1+\xi(x))^{\alpha}\right]^{\prime}=\frac{\alpha}{(1+\xi(x))^{1-\alpha}} \xi^{\prime}(x) \leq \alpha \xi^{\prime}(x)=[1+\alpha \xi(x)]^{\prime} .
$$

LEMMA 4.2. For $x \in I \backslash \Delta$, we have $g(x) \leq 1+C_{0} x^{2 \alpha}$, where

$$
C_{0}=\frac{\alpha(1+\alpha)}{2}\left[1+2 \delta_{0}\left(x_{0}\right)+\delta_{0}^{2}\left(x_{0}\right)\right] .
$$

Proof. Using Lemma 4.1, we have

$$
\begin{aligned}
g(x) & =\frac{\left(T_{1} x / x\right)^{1+\alpha}}{T_{1}^{\prime}(x)}=\frac{\left[1+x^{\alpha}+x^{\alpha} \delta_{0}(x)\right]^{1+\alpha}}{1+(1+\alpha) x^{\alpha}+x^{\alpha} \delta_{1}(x)} \\
& \leq \frac{1+(1+\alpha)\left[x^{\alpha}+x^{\alpha} \delta_{0}(x)\right]+(\alpha(1+\alpha) / 2)\left[x^{\alpha}+x^{\alpha} \delta_{0}(x)\right]^{2}}{1+(1+\alpha) x^{\alpha}+x^{\alpha} \delta_{1}(x)} \\
& =\frac{1+(1+\alpha)\left[x^{\alpha}+x^{\alpha} \delta_{0}(x)\right]}{1+(1+\alpha) x^{\alpha}+x^{\alpha} \delta_{1}(x)}+\frac{(\alpha(1+\alpha) / 2)\left[x^{\alpha}+x^{\alpha} \delta_{0}(x)\right]^{2}}{1+(1+\alpha) x^{\alpha}+x^{\alpha} \delta_{1}(x)} \\
& \leq 1+\frac{\alpha(1+\alpha)}{2}\left[x^{\alpha}+x^{\alpha} \delta_{0}(x)\right]^{2} \\
& =1+\frac{\alpha(1+\alpha)}{2}\left(1+2 \delta_{0}(x)+\delta_{0}^{2}(x)\right) x^{2 \alpha} \leq 1+C_{0} x^{2 \alpha} .
\end{aligned}
$$

LEMMA 4.3. Let $x_{n}=T_{1}^{-n} x_{0}$. For $n \geq 1$, we have $x_{n} \leq C_{1} n^{-1 / \alpha}$, where $C_{1}=\left(2\left[2^{1 / \alpha}-\right.\right.$ $1])^{1 / \alpha}$.

$\dagger$ It is obvious that $\xi(0)=0$ and for $x>0, \xi(x)>0$. 
Proof. Observe that $C_{1}>1 \geq T_{1}^{-1}\left(x_{0}\right)=x_{1}$. Therefore, the lemma is true for $n=1$. Next, for $n \geq 2$, we suppose that $x_{n-1} \leq C_{1}(n-1)^{-1 / \alpha}$, and prove that $x_{n} \leq C_{1} n^{-1 / \alpha}$. If it is false, that is $x_{n}>C_{1} n^{-1 / \alpha}$, then by our inductive statement on $x_{n-1}$, we have

$$
C_{1}(n-1)^{-1 / \alpha} \geq x_{n-1}=T_{1}\left(x_{n}\right)>C_{1} n^{-1 / \alpha}\left[1+C_{1}^{\alpha} n^{-1}+C_{1}^{\alpha} n^{-1} \delta_{0}\left(C_{1} n^{-1 / \alpha}\right)\right] .
$$

This is equivalent to

$$
n\left[\left(1+\frac{1}{n-1}\right)^{1 / \alpha}-1\right]>C_{1}^{\alpha}\left[1+\delta_{0}\left(C_{1} n^{-1 / \alpha}\right)\right] .
$$

By convexity of the function $z^{1 / \alpha}$,

$$
\frac{n}{n-1}\left[2^{1 / \alpha}-1\right]>C_{1}^{\alpha}\left[1+\delta_{0}\left(C_{1} n^{-1 / \alpha}\right)\right]
$$

that is,

$$
C_{1}^{\alpha}<\frac{n}{n-1}\left[2^{1 / \alpha}-1\right] /\left[1+\delta_{0}\left(C_{1} n^{-1 / \alpha}\right)\right]<2\left[2^{1 / \alpha}-1\right]=C_{1}^{\alpha} .
$$

This represents a contradiction, and therefore, $x_{n} \leq C_{1} n^{-1 / \alpha}$. This completes the proof of the lemma.

LEMMA 4.4. For $x \in I \backslash \Delta$, we have

$$
G_{1}(x) \leq \frac{x_{0}^{1+\alpha}}{\beta}
$$

and for $n \geq 2$,

$$
G_{n}(x) \leq M(n-1)^{-(1+(1 / \alpha))},
$$

where $M=\left(C_{1}^{1+\alpha} e^{2 C_{0} C_{1}^{2 \alpha}}\right) / \beta$.

Proof. For $n=1$, it is easy to see that

$$
G_{1}(x) \leq \frac{x_{0}^{1+\alpha}}{\beta} .
$$

For $n \geq 2$, we have

$$
\begin{aligned}
G_{n}(x)= & \frac{x^{1+\alpha}}{\left|D T^{(n)}\left(T_{2}^{-1} T_{1}^{-(n-1)} x\right)\right|} \\
= & \frac{x^{1+\alpha}}{\left|D\left(T_{1} \circ T_{1} \circ \cdots \circ T_{1} \circ T_{2}\right)\left(T_{2}^{-1} T_{1}^{-(n-1)} x\right)\right|} \\
= & \frac{x^{1+\alpha}}{T_{1}^{\prime}\left(T_{1}^{-1} x\right) \cdot T_{1}^{\prime}\left(T_{1}^{-2} x\right) \cdots T_{1}^{\prime}\left(T_{1}^{-(n-1)} x\right) \cdot\left|T_{2}^{\prime}\left(T_{2}^{-1} T_{1}^{-(n-1)} x\right)\right|} \\
= & \frac{\left(x / T_{1}^{-1} x\right)^{1+\alpha}}{T_{1}^{\prime}\left(T_{1}^{-1} x\right)} \cdot \frac{\left(T_{1}^{-1} x / T_{1}^{-2} x\right)^{1+\alpha}}{T_{1}^{\prime}\left(T_{1}^{-2} x\right)} \cdots \frac{\left(T_{1}^{-(n-2)} x / T_{1}^{-(n-1)} x\right)^{1+\alpha}}{T_{1}^{\prime}\left(T_{1}^{-(n-1)} x\right)} \\
& \cdot \frac{\left(T_{1}^{-(n-1)} x\right)^{1+\alpha}}{\left|T_{2}^{\prime}\left(T_{2}^{-1} T_{1}^{-(n-1)} x\right)\right|}
\end{aligned}
$$




$$
\begin{aligned}
& =g\left(T_{1}^{-1} x\right) \cdot g\left(T_{1}^{-2} x\right) \cdots g\left(T_{1}^{-(n-1)} x\right) \cdot \frac{\left(T_{1}^{-(n-1)} x\right)^{1+\alpha}}{\left|T_{2}^{\prime}\left(T_{2}^{-1} T_{1}^{-(n-1)} x\right)\right|} \\
& \leq g\left(T_{1}^{-1} x\right) \cdot g\left(T_{1}^{-2} x\right) \cdots g\left(T_{1}^{-(n-1)} x\right) \cdot \frac{\left(T_{1}^{-(n-1)} x\right)^{1+\alpha}}{\beta} .
\end{aligned}
$$

By Lemmas 4.2 and 4.3 , for any $k \geq 1, x \in\left[0, x_{0}\right)$, we have

$$
\begin{aligned}
g\left(T_{1}^{-k}(x)\right) & \leq 1+C_{0}\left(T_{1}^{-k}(x)\right)^{2 \alpha} \leq 1+C_{0}\left(T_{1}^{-k}\left(x_{0}\right)\right)^{2 \alpha} \\
& =1+C_{0}\left(x_{k}\right)^{2 \alpha} \leq 1+C_{0} C_{1}^{2 \alpha} k^{-2} .
\end{aligned}
$$

Therefore, using (4.2) and (4.3), for $n \geq 2$, we obtain

$$
\begin{aligned}
G_{n}(x) & \leq \prod_{k=1}^{n-1} g\left(T_{1}^{-k}(x)\right) \cdot \frac{\left(T_{1}^{-(n-1)}(x)\right)^{1+\alpha}}{\beta} \\
& \leq \prod_{k=1}^{n-1}\left(1+C_{0} C_{1}^{2 \alpha} k^{-2}\right) \cdot \frac{C_{1}^{1+\alpha}(n-1)^{-(1+(1 / \alpha))}}{\beta} \\
& =\exp \left\{\sum_{k=1}^{n-1} \ln \left(1+C_{0} C_{1}^{2 \alpha} k^{-2}\right)\right\} \cdot \frac{C_{1}^{1+\alpha}(n-1)^{-(1+(1 / \alpha))}}{\beta} \\
& \leq \exp \left\{\sum_{k=1}^{n-1} C_{0} C_{1}^{2 \alpha} k^{-2}\right\} \cdot \frac{C_{1}^{1+\alpha}(n-1)^{-(1+(1 / \alpha))}}{\beta} \\
& \leq \exp \left\{C_{0} C_{1}^{2 \alpha}\left(2-\frac{1}{n-1}\right)\right\} \cdot \frac{C_{1}^{1+\alpha}(n-1)^{-(1+(1 / \alpha))}}{\beta} \\
& \leq M(n-1)^{-(1+(1 / \alpha))} .
\end{aligned}
$$

Finally we estimate the difference between the normalizing constants appearing in equations (3.2) and (3.3).

LEMMA 4.5.

$$
\sum_{n=1}^{\infty} n \cdot \hat{\lambda}\left(Z_{n}\right) \leq C_{3}
$$

where

$$
C_{3}=\frac{1}{\beta}+\frac{C_{2}}{\beta\left(1-x_{0}\right)}\left(\alpha+\frac{2-\alpha}{1-\alpha}\right)
$$

and

$$
C_{2}=\frac{1-x_{0}}{x_{0}^{1+\alpha}} 2^{1+(1 / \alpha)}\left[2^{1 / \alpha}-1\right]^{1+(1 / \alpha)} .
$$

Proof. By Lemma 4.3, we have

$$
\begin{aligned}
\lambda\left(W_{n}\right) & =x_{n-1}-x_{n}=T_{1}\left(x_{n}\right)-x_{n}=\frac{1-x_{0}}{x_{0}^{1+\alpha}} x_{n}^{1+\alpha} \\
& \leq \frac{1-x_{0}}{x_{0}^{1+\alpha}} C_{1}^{1+\alpha} n^{-(1+(1 / \alpha))}=C_{2} n^{-(1+(1 / \alpha))} .
\end{aligned}
$$


Since $T_{2}\left(Z_{n}\right)=W_{n-1}$, we have

$$
\begin{aligned}
\sum_{n=1}^{\infty} n \cdot \lambda\left(Z_{n}\right) & \leq \lambda\left(Z_{1}\right)+\sum_{n=2}^{\infty} n \cdot \frac{\lambda\left(W_{n-1}\right)}{\beta} \\
& \leq \frac{1-x_{0}}{\beta}+\sum_{n=2}^{\infty} \frac{n\left(x_{n-2}-x_{n-1}\right)}{\beta} \\
& =\frac{1-x_{0}}{\beta}+\sum_{n=1}^{\infty} \frac{(n+1)\left(x_{n-1}-x_{n}\right)}{\beta} \\
& =\frac{1-x_{0}}{\beta}+\sum_{n=1}^{\infty} \frac{n\left(x_{n-1}-x_{n}\right)}{\beta}+\sum_{n=1}^{\infty} \frac{\left(x_{n-1}-x_{n}\right)}{\beta} \\
& \leq \frac{1-x_{0}}{\beta}+\sum_{n=1}^{\infty} \frac{C_{2}}{\beta} n^{-1 / \alpha}+\sum_{n=1}^{\infty} \frac{C_{2}}{\beta} n^{-(1+(1 / \alpha))} \\
& \leq \frac{1-x_{0}}{\beta}+\frac{C_{2}}{\beta}\left(1+\int_{1}^{\infty} x^{-1 / \alpha} d x\right)+\frac{C_{2}}{\beta}\left(1+\int_{1}^{\infty} x^{-(1+(1 / \alpha))} d x\right) \\
& =\frac{1-x_{0}}{\beta}+\frac{C_{2}}{\beta}\left(\alpha+\frac{2-\alpha}{1-\alpha}\right)=\left(1-x_{0}\right) \cdot C_{3} .
\end{aligned}
$$

This completes the proof of the lemma since $\hat{\lambda}(\cdot)=\lambda(\cdot) /\left(1-x_{0}\right)$.

LEMMA 4.6. We have

$$
\left|c_{\tau, m}-c_{\tau}\right| \leq C_{3} \cdot \hat{C} \frac{\ln m}{m}
$$

Proof. Using the fact that $c_{\tau} \leq 1, c_{\tau, m} \leq 1$ and Theorem 2.1, we have

$$
\begin{aligned}
\left|c_{\tau, m}-c_{\tau}\right| & \leq\left|\frac{1}{\sum_{k=1}^{\infty} \tau_{Z_{k}} \hat{\mu}_{m}\left(Z_{k}\right)}-\frac{1}{\sum_{k=1}^{\infty} \tau_{Z_{k}} \hat{\mu}\left(Z_{k}\right)}\right| \\
& =\left|\frac{\sum_{k=1}^{\infty} k\left[\hat{\mu}\left(Z_{k}\right)-\hat{\mu}_{m}\left(Z_{k}\right)\right]}{\sum_{k=1}^{\infty} \tau_{Z_{k}} \hat{\mu}_{m}\left(Z_{k}\right) \cdot \sum_{k=1}^{\infty} \tau_{Z_{k}} \hat{\mu}\left(Z_{k}\right)}\right| \\
& \leq\left(\sum_{k=1}^{\infty} k \int_{Z_{k}}\left|\hat{f}-\hat{f}_{m}\right| d \hat{\lambda}\right) \\
& \leq\left\|\hat{f}-\hat{f}_{m}\right\|_{\infty}\left(\sum_{k=1}^{\infty} k \hat{\lambda}\left(Z_{k}\right)\right) \\
& \leq \hat{C} \frac{\ln m}{m} \cdot C_{3} .
\end{aligned}
$$

In the last estimate, we have used Lemma 4.5.

We now have all our tools ready to prove Theorem 3.1. 
Proof of Theorem 3.1. Using (3.2) and (3.3), we have

$$
\begin{aligned}
\left\|f^{*}-f_{m}\right\|_{\mathcal{B}}= & \sup _{x \in(0,1]}\left|x^{1+\alpha}\left(f^{*}(x)-f_{m}(x)\right)\right| \\
\leq & \sup _{x \in I \backslash \Delta}\left|x^{1+\alpha}\left(f^{*}(x)-f_{m}(x)\right)\right|+\sup _{x \in \Delta}\left|x^{1+\alpha}\left(f^{*}(x)-f_{m}(x)\right)\right| \\
= & \sup _{x \in I \backslash \Delta} \mid \sum_{n=1}^{\infty} \frac{x^{1+\alpha}}{D T^{(n)}\left(T_{2}^{-1} T_{1}^{-(n-1)} x\right)}\left(c_{\tau} \hat{f}\left(T_{2}^{-1} T_{1}^{-(n-1)} x\right)\right. \\
& \left.-c_{\tau, m} \hat{f}_{m}\left(T_{2}^{-1} T_{1}^{-(n-1)} x\right)\right) \mid \\
& +\sup _{x \in \Delta}\left|x^{1+\alpha}\left(c_{\tau} \hat{f}(x)-c_{\tau, m} \hat{f}_{m}(x)\right)\right| .
\end{aligned}
$$

Notice that for $x \in I \backslash \Delta$, and $n \geq 1, z_{n}:=T_{2}^{-1} T_{1}^{-(n-1)} x \in \Delta$. Then using the fact that $c_{\tau} \leq 1, c_{\tau, m} \leq 1$, Theorem 2.1, Lemma 4.6, and (4.4), we obtain

$$
\begin{aligned}
\left\|f^{*}-f_{m}\right\|_{\mathcal{B}} \leq & \sup _{x \in I \backslash \Delta}\left|\sum_{n=1}^{\infty} \frac{x^{1+\alpha}}{D T^{(n)}\left(T_{2}^{-1} T_{1}^{-(n-1)} x\right)}\right| \cdot \sup _{z_{n} \in \Delta} \mid\left(c_{\tau} \hat{f}\left(z_{n}\right)-c_{\tau, m} \hat{f}_{m}\left(z_{n}\right) \mid\right. \\
& +\sup _{x \in \Delta}\left|c_{\tau} \hat{f}(x)-c_{\tau, m} \hat{f}_{m}(x)\right| \\
\leq & \sup _{x \in I \backslash \Delta}\left|\sum_{n=1}^{\infty} \frac{x^{1+\alpha}}{D T^{(n)}\left(T_{2}^{-1} T_{1}^{-(n-1)} x\right)}\right| \\
& \times\left(\sup _{z_{n} \in \Delta}\left|\hat{f}\left(z_{n}\right)-\hat{f}_{m}\left(z_{n}\right)\right|+\left|c_{\tau}-c_{\tau, m}\right| \sup _{z_{n} \in \Delta}\left|\hat{f}\left(z_{n}\right)\right|\right) \\
& +\sup _{x \in \Delta}\left|\hat{f}(x)-\hat{f}_{m}(x)\right|+\left|c_{\tau}-c_{\tau, m}\right| \sup _{x \in \Delta}|\hat{f}(x)| \\
\leq & \hat{C} \frac{\ln m}{m}\left(\sup _{x \in I \backslash \Delta} \sum_{n=1}^{\infty}\left|G_{n}(x)\right|\left(1+C_{3} \sup _{z_{n} \in \Delta}\left|\hat{f}\left(z_{n}\right)\right|\right)+\left(1+C_{3} \sup _{x \in \Delta}|\hat{f}(x)|\right)\right) .
\end{aligned}
$$

Since $\hat{f} \in B V(\Delta)$, we have $\sup _{x \in \Delta}|\hat{f}(x)| \leq V_{\Delta} \hat{f}+\left(1 /\left(1-x_{0}\right)\right)\|\hat{f}\|_{1, \Delta}$. Therefore, using the Lasota-Yorke inequality (2.1), we obtain $\sup _{x \in \Delta}|\hat{f}(x)| \leq C_{\mathrm{LY}} /(1-\gamma)+$ $1 /\left(1-x_{0}\right)$. Using Lemma 4.4 and (4.5), we obtain

$$
\begin{aligned}
\left\|f^{*}-f_{m}\right\|_{\mathcal{B}} & \leq C_{4} \hat{C} \frac{\ln m}{m}\left(1+\frac{x_{0}^{1+\alpha}}{\beta}+\sum_{n=2}^{\infty} M(n-1)^{-(1+(1 / \alpha))}\right) \\
& =C_{4} \hat{C} \frac{\ln m}{m}\left(1+\frac{x_{0}^{1+\alpha}}{\beta}+M \sum_{n=1}^{\infty} n^{-(1+(1 / \alpha))}\right) \\
& \leq C_{4} \hat{C}\left(1+\frac{x_{0}^{1+\alpha}}{\beta}+M(1+\alpha)\right) \cdot \frac{\ln m}{m} .
\end{aligned}
$$

\section{Algorithm and feasibility}

Given a map $T$ satisfying the conditions of $\S 3.1$, and $x^{*} \in(0,1]$, we provide an algorithm based on Corollary 3.2 that can be used to approximate $f^{*}\left(x^{*}\right)$, the $T$-invariant density at the point $x^{*}$, up to a pre-specified approximation error $R$. 


\subsection{Algorithm and output.}

(1) Compute the constants $\beta, M, C_{4}$ which appear in Theorem 3.1.

(2) Compute an upper bound on the constant $\hat{C}$ which appears in Theorem 2.1.

(3) Then use (1), (2) to compute $C^{*}$ which appears in Theorem 3.1.

(4) Find $m^{*}$, number of bins, such that

$$
\frac{\ln m^{*}}{m^{*}} \leq \frac{x^{* 1+\alpha}}{C^{*}} \cdot \frac{R}{3}
$$

(5) Compute an approximate fixed point $\dagger$. $\tilde{\hat{f}}_{m}>0$ of $\mathbb{P}_{m}$ so that $\left\|\tilde{\hat{f}}_{m}-\hat{f}_{m}\right\|_{\infty} \leq \varepsilon$ on $\Delta$, where $\varepsilon$ is chosen such that

$$
\left|\tilde{f}_{m}\left(x^{*}\right)-f_{m}\left(x^{*}\right)\right| \leq \frac{R}{3}
$$

where

$$
\tilde{f}_{m}(x)=\tilde{c}_{\tau, m} \sum_{n=1}^{\infty}\left(\frac{\tilde{\hat{f}}_{m}\left(T_{2}^{-1} T_{1}^{-(n-1)} x\right)}{\left|D T^{(n)}\left(T_{2}^{-1} T_{1}^{-(n-1)} x\right)\right|}\right),
$$

$\tilde{c}_{\tau, m}^{-1}:=\sum_{k=1}^{\infty} k \tilde{\hat{\mu}}_{m}\left(Z_{k}\right)$ and $\tilde{\hat{\mu}}=\tilde{\hat{f}}_{m} \cdot \hat{\lambda}$.

(6) Find $N^{*}$ such that

$$
\left|\tilde{c}_{\tau, m}\left(N^{*}\right) \sum_{n=1}^{N^{*}}\left(\frac{\tilde{\hat{f}}_{m}\left(T_{2}^{-1} T_{1}^{-(n-1)} x^{*}\right)}{\left|D T^{(n)}\left(T_{2}^{-1} T_{1}^{-(n-1)} x^{*}\right)\right|}\right)-\tilde{f}_{m}\left(x^{*}\right)\right| \leq \frac{R}{3},
$$

where $\tilde{c}_{\tau, m}^{-1}\left(N^{*}\right):=\sum_{k=1}^{N^{*}} k \tilde{\hat{\mu}}_{m}\left(Z_{k}\right)$.

(7) The approximate value of $f^{*}\left(x^{*}\right)$ is given by $\ddagger$ :

$$
\tilde{f}_{m, N^{*}}\left(x^{*}\right):=\tilde{c}_{\tau, m}\left(N^{*}\right) \sum_{n=1}^{N^{*}}\left(\frac{\tilde{\hat{f}}_{m}\left(T_{2}^{-1} T_{1}^{-(n-1)} x^{*}\right)}{\left|D T^{(n)}\left(T_{2}^{-1} T_{1}^{-(n-1)} x^{*}\right)\right|}\right) .
$$

\subsection{Feasibility.}

- For (1), once the map $T$ is given, the constants $\beta, M, C_{4}$ can be computed analytically.

- $\quad$ For (2), the constant $\hat{C}$ appears in the approximation done on the induced system (see Theorem 2.1). The induced system is a uniformly expanding map. The computation of $\hat{C}$ can be done following the ideas of [2], which is based on the spectral stability result of [16].

- (3) is a consequence of (1) and (2).

$\dagger$ Recall that $\hat{f}_{m}$ is the fixed point of the finite rank operator $\mathbb{P}_{m}$ defined in (2.3). Here $\tilde{\hat{f}}_{m}$ is the computer approximation of $\hat{f}_{m}$, i.e. (5) of the Algorithm takes care of the computer roundoff errors in computing the fixed point of $\mathbb{P}_{m}$. Since $\hat{f}_{m}>0$, we also ask in this computation that $\tilde{\hat{f}}_{m}>0$. Note that the strict positivity of $\hat{f}_{m}$ follows from the fact that the induced map $\hat{T}$ is a piecewise onto map, which implies that the matrix representation of $\mathbb{P}$ is irreducible, and consequently its Perron eigenvector is strictly positive (see PerronFrobenius theorem [17]).

$\$$ It is very important to notice that the approximation $\tilde{f}_{m, N^{*}}\left(x^{*}\right)$ is a finite sum. 
- $\quad$ Once $C^{*}$ is computed in (3), with $m:=m^{*}$ we know, from Corollary 3.2, that $\left|f_{m}\left(x^{*}\right)-f^{*}\left(x^{*}\right)\right| \leq R / 3$.

- $\quad$ For (5) we should find out how small $\varepsilon$ should be to ensure

$$
\left|\tilde{f}_{m}\left(x^{*}\right)-f_{m}\left(x^{*}\right)\right| \leq \frac{R}{3} .
$$

We propose the following method. To work out explicitly all the constants needed in verifying (5), we suppose $x^{*} \in W_{k}$, and $T_{1}(x)=x+2^{\alpha} x^{1+\alpha}$. In the following estimates (5.2), (5.3) and (5.4), we prepare the ingredients to achieve our job. Firstly, following exactly the argument of [3, Lemma 5.2], for $x^{*} \in W_{k}$,

$$
\left|D T^{(n)}\left(T_{2}^{-1} T_{1}^{-(n-1)} x^{*}\right)\right| \geq \beta\left(\frac{n+k}{k+2}\right)^{\eta_{k}},
$$

where

$$
\eta_{k}=\frac{d(k+2)}{k+2+d}, \quad d=(1+\alpha) 2^{\alpha}\left[\frac{1}{2(1+\alpha)^{1 / \alpha}}+d_{1}\right]^{\alpha}>1
$$

and

$$
d_{1}=\frac{1}{2(\alpha)^{1 / \alpha}}-\frac{1}{2(1+\alpha)^{1 / \alpha}} .
$$

Consequently, $\eta_{k}>1$, and for $x^{*} \in W_{k}$,

$$
\sum_{n=1}^{\infty} \frac{1}{\left|D T^{(n)}\left(T_{2}^{-1} T_{1}^{-(n-1)} x^{*}\right)\right|} \leq \frac{1}{\beta}\left(\frac{k+2}{k}\right)^{\eta_{k}} \frac{k}{\eta_{k}-1} .
$$

Secondly, using the same argument as that in the proof of Lemma 4.6, we have

$$
\left|\tilde{c}_{\tau, m}-c_{\tau, m}\right| \leq C_{3} \cdot\left\|\tilde{\hat{f}}_{m}-\hat{f}_{m}\right\|_{\infty} .
$$

Thirdly, it is well known $\dagger$ that for $g \in B V(\Delta)$ we have $V_{\Delta} Q_{m} g \leq g$, where $Q_{m}$ is the discretization defined in $\$ 2.2$. Therefore, $\mathbb{P}_{m}$ satisfies the same Lasota-Yorke inequality (2.1) as $\hat{\mathcal{L}}$. In particular, this implies that $\hat{f}_{m}$, the $\mathbb{P}_{m}$ fixed point, satisfies:

$$
\left\|\hat{f}_{m}\right\|_{\infty} \leq \frac{C_{\mathrm{LY}}}{1-\gamma}+\frac{1}{|\Delta|}
$$

Consequently, using (5.2)-(5.4) and that $\tilde{c}_{\tau, m} \leq 1$, we obtain

$$
\begin{aligned}
& \left|\tilde{f}_{m}\left(x^{*}\right)-f_{m}\left(x^{*}\right)\right| \\
& \quad \leq\left\|\tilde{\hat{f}}_{m}-\hat{f}_{m}\right\|_{\infty} \cdot \frac{1}{\beta}\left(\frac{k+2}{k}\right)^{\eta_{k}} \frac{k}{\eta_{k}-1}\left[1+C_{3}\left(\frac{C_{\mathrm{LY}}}{1-\gamma}+\frac{1}{|\Delta|}\right)\right] .
\end{aligned}
$$

Thus, to ensure $\left|\tilde{f}_{m}\left(x^{*}\right)-f_{m}\left(x^{*}\right)\right| \leq R / 3, \tilde{\hat{f}}_{m}$ should be computed to a precision that satisfies

$$
\begin{aligned}
& \left\|\tilde{\hat{f}}_{m}-\hat{f}_{m}\right\|_{\infty} \\
& \quad \leq \frac{1}{(1 / \beta)((k+2) / k)^{\eta_{k}}\left(k /\left(\eta_{k}-1\right)\right)\left[1+C_{3}\left(C_{\mathrm{LY}} /(1-\gamma)+1 /|\Delta|\right)\right]} \cdot \frac{R}{3} .
\end{aligned}
$$

$\dagger$ See, for instance, [9, Lemma 2.3] 
- $\quad$ For (6), as in (5), we also suppose $x^{*} \in W_{k}$, and $T_{1}(x)=x+2^{\alpha} x^{1+\alpha}$. Firstly, by (5.1), each term in the sum

$$
\sum_{n=1}^{\infty}\left(\frac{\tilde{\hat{f}}_{m}\left(T_{2}^{-1} T_{1}^{-(n-1)} x^{*}\right)}{\left|D T^{(n)}\left(T_{2}^{-1} T_{1}^{-(n-1)} x^{*}\right)\right|}\right)
$$

is bounded above by

$$
\sup _{y \in \Delta} \tilde{\hat{f}}_{m}(y) \cdot \frac{1}{\beta}\left(\frac{k+2}{n+k}\right)^{\eta_{k}} .
$$

Since $\eta_{k}>1$, the tail of the sum, starting from $N_{1}^{*}+1$, in (5.6) can be approximated as follows.

Choose $N_{1}^{*}$ such that

$$
\sup _{y \in \Delta} \tilde{\hat{f}}_{m}(y) \cdot \frac{\left(N_{1}^{*}+k\right)^{-\eta_{k}+1}}{\eta_{k}-1} \cdot(k+2)^{\eta_{k}} \leq \frac{R}{6} .
$$

Secondly, using $\sum_{k=1}^{\infty} k \tilde{\hat{\mu}}_{m}\left(Z_{k}\right) \geq 1$, and recalling that $\alpha \in(0,1)$ and $\inf _{y \in \Delta}$ $\tilde{\hat{f}}_{m}(y)>0$, we have

$$
\begin{aligned}
\left|\tilde{c}_{\tau, m}-\tilde{c}_{\tau, m}\left(N_{2}^{*}\right)\right| & =\frac{\sum_{N_{2}^{*}+1}^{\infty} k \tilde{\hat{\mu}}_{m}\left(Z_{k}\right)}{\sum_{k=1}^{\infty} k \tilde{\hat{\mu}}_{m}\left(Z_{k}\right) \cdot \sum_{k=1}^{N_{2}^{*}} k \tilde{\hat{\mu}}_{m}\left(Z_{k}\right)} \\
& \leq \sup _{y \in \Delta} \tilde{\hat{f}}_{m} \frac{\sum_{N_{2}^{*}+1}^{\infty} k \hat{\lambda}\left(Z_{k}\right)}{\sum_{k=1}^{N_{2}^{*}} k \tilde{\hat{\mu}}_{m}\left(Z_{k}\right)} \\
& \leq \sup _{y \in \Delta} \tilde{\hat{f}}_{m} \frac{\sum_{N_{2}^{*}+1}^{\infty}\left(C_{2} / \beta\right) \cdot k \cdot k^{-1-(1 / \alpha)}}{\inf _{y \in \Delta} \tilde{\hat{f}}_{m} \cdot \hat{\lambda}\left(Z_{1}\right)} \\
& \leq \frac{\sup _{y \in \Delta} \tilde{\hat{f}}_{m}}{\inf _{y \in \Delta} \tilde{\hat{f}}_{m} \cdot \hat{\lambda}\left(Z_{1}\right)} \frac{C_{2}}{\beta} \cdot \frac{N_{2}^{*}(-1 / \alpha)+1}{(1 / \alpha)-1} .
\end{aligned}
$$

Thirdly, using (5.2) and (5.8), we have

$$
\begin{aligned}
& \left|\tilde{c}_{\tau, m}-\tilde{c}_{\tau, m}\left(N_{2}^{*}\right)\right| \tilde{f}_{m, N_{2}^{*}}\left(x^{*}\right) \\
& \quad \leq\left(\frac{\sup _{y \in \Delta} \tilde{\hat{f}}_{m}}{\inf _{y \in \Delta} \tilde{\hat{f}}_{m} \cdot \hat{\lambda}\left(Z_{1}\right)} \frac{C_{2}}{\beta} \cdot \frac{N_{2}^{*(-1 / \alpha)+1}}{(1 / \alpha)-1}\right) \tilde{f}_{m}\left(x^{*}\right) \\
& \quad \leq \frac{\sup _{y \in \Delta} \tilde{\hat{f}}_{m}^{2}}{\inf _{y \in \Delta} \tilde{\hat{f}}_{m} \cdot \hat{\lambda}\left(Z_{1}\right)} \frac{C_{2}}{\beta^{2}} \cdot \frac{N_{2}^{*(-1 / \alpha)+1}}{(1 / \alpha)-1}\left(\frac{k+2}{k}\right)^{\eta_{k}} \frac{k}{\eta_{k}-1} .
\end{aligned}
$$

Choose $\dagger N_{2}^{*}$ such that the right-hand side of (5.9) is not greater than $R / 6$. Finally, choose $N^{*}=\max \left\{N_{1}^{*}, N_{2}^{*}\right\}$. This will lead to the desired estimate $\mid \tilde{f}_{m}\left(x^{*}\right)-$ $\tilde{f}_{m, N^{*}}\left(x^{*}\right) \mid \leq R / 3$.

$\dagger$ In our analysis we verified many items of the Algorithm analytically. Many of these steps may be verified iteratively using a computer. For instance, $N_{2}^{*}$ may be found iteratively using a computer. Simply keep $\sum_{k=1}^{N_{2}^{*}} k \tilde{\hat{\mu}}_{m}\left(Z_{k}\right)$ in the denominator on the right-hand side of (5.9) instead of replacing it by inf $\operatorname{in}_{y \in \Delta} \tilde{\hat{f}}_{m} \cdot \hat{\lambda}\left(Z_{1}\right)$. The iterative method may find a smaller value of $N_{2}^{*}$ that achieves the job. 
- $\quad$ For (7), using the above six steps, we obtain

$$
\begin{aligned}
\left|f^{*}\left(x^{*}\right)-\tilde{f}_{m, N^{*}}\left(x^{*}\right)\right| \leq & \left|f^{*}\left(x^{*}\right)-f_{m}\left(x^{*}\right)\right|+\mid f_{m}\left(x^{*}\right) \\
& -\tilde{f}_{m}\left(x^{*}\right)|+| \tilde{f}_{m}\left(x^{*}\right)-\tilde{f}_{m, N^{*}}\left(x^{*}\right) \mid \leq R
\end{aligned}
$$

i.e. the finite sum

$$
\tilde{f}_{m, N^{*}}\left(x^{*}\right):=\tilde{c}_{\tau, m}\left(N^{*}\right) \sum_{n=1}^{N^{*}}\left(\frac{\tilde{\hat{f}}_{m}\left(T_{2}^{-1} T_{1}^{-(n-1)} x^{*}\right)}{\left|D T^{(n)}\left(T_{2}^{-1} T_{1}^{-(n-1)} x^{*}\right)\right|}\right)
$$

is a rigorous approximation of $f^{*}\left(x^{*}\right)$ up to the pre-specified error $R$.

Acknowledgement. The authors would like to thank an anonymous referee for comments that improved both the presentation and the content of the paper.

\section{REFERENCES}

[1] H. Aytac, J. Freitas and S. Vaienti. Laws of rare events for deterministic and random dynamical systems. Preprint, 2012, arxiv.org/pdf/1207.5188.

[2] W. Bahsoun and C. Bose. Invariant densities and escape rates: rigorous and computable approximations in the $L^{\infty}$-norm. Nonlinear Anal. 74 (2011), 4481-4495.

[3] W. Bahsoun and S. Vaienti. Metastability of certain intermittent maps. Nonlinearity 25(1) (2012), 107124.

[4] V. Baladi. Positive Transfer Operators and Decay of Correlations (Advanced Series in Nonlinear Dynamics, 16). World Science Publications, River Edge, NJ, 2000.

[5] M. Blank. Finite rank approximations of expanding maps with neutral singularities. Discrete Contin. Dyn. Syst. 21(3) (2008), 749-762.

[6] M. Blank, G. Keller and C. Liverani. Ruelle-Perron-Frobenius spectrum for Anosov maps. Nonlinearity 15(6) (2002), 1905-1973.

[7] C. Bose. Invariant measures and equilibrium states for piecewise $C^{1+\alpha}$ endomorphisms of the unit interval. Trans. Amer. Math. Soc. 315(1) (1989), 105-125.

[8] A. Boyarsky and P. Góra. Laws of Chaos: Invariant Measures and Dynamical Systems in one Dimension. Birkhäuser, Boston, 1997.

[9] J. Ding and T. A. Li. Convergence rate analysis for Markov approximations to a class of Frobenius-Perron operators. Nonlinear Anal. 31 (1998), 765-777.

[10] G. Froyland. Finite approximation of Sinai-Bowen-Ruelle measures for Anosov systems in two dimensions. Random Comput. Dynam. 3(4) (1995), 251-263.

[11] G. Froyland. On Ulam approximation of the isolated spectrum and eigenfunctions of hyperbolic maps. Discrete Contin. Dyn. Syst. 17(3) (2007), 671-689.

[12] S. Galatolo and I. Nisoli. An elementary approach to rigorous approximation of invariant measures. Preprint, 2011, arXiv:1109.2342.

[13] M. Holland, M. Nicol and A. Török. Extreme value theory for non-uniformly expanding dynamical systems. Trans. Amer. Math. Soc. 364(2) (2012), 661-688.

[14] H. Hu. Decay of correlations for piecewise smooth maps with indifferent fixed points. Ergod. Th. \& Dynam. Sys. 24(2) (2004), 495-524.

[15] G. Keller. Rare events, exponential hitting times and extremal indices via spectral perturbation. Dyn. Syst. 27(1) (2012), 11-27.

[16] G. Keller and C. Liverani. Stability of the spectrum for transfer operators. Ann. Sc. Norm. Super. Pisa Cl. Sci. (4) 28(1) (1999), 141-152.

[17] P. Lancaster and M. Tismenetsky. The Theory of Matrices. Academic Press, Orlando, FL, 1985.

[18] C. Liverani. Rigorous numerical investigation of the statistical properties of piecewise expanding maps. A feasibility study. Nonlinearity 14(3) (2001), 463-490.

[19] C. Liverani, B. Saussol and S. Vaienti. A probabilistic approach to intermittency. Ergod. Th. \& Dynam. Sys. 19 (1999), 671-685. 
[20] R. Murray. Existence, mixing and approximation of invariant densities for expanding maps on $R^{r}$. Nonlinear Anal. 45(1) (2001), 37-72.

[21] R. Murray. Ulam's method for some non-uniformly expanding maps. Discrete. Contin. Dyn. Syst. 26(3) (2010), 1007-1018.

[22] G. Pianigiani. First return map and invariant measures. Israel J. Math. 35 (1980), 32-48.

[23] S. M. Ulam. A Collection of Mathematical Problems (Interscience Tracts in Pure and Applied Mathematics, 8). Interscience, New York, 1960.

[24] L.-S. Young. Recurrence times and rates of mixing. Israel J. Math. 110 (1999), 153-188. 